\title{
Equilibre entre les apports azotés et énergétiques dans l'alimentation du Lapin en croissance
}

\author{
Chantal DEHALLE \\ I.N.R.A., Laboratoire de Recherches sur l'Elevage du Lapin \\ Centre de Recherches de Toulouse, B.P. 12, 31320 Castanet-Tolosan (France)
}

\begin{abstract}
Résumé
Soixante lapins des deux sexes âgés de 5 semaines ont été alimentés à volonté avec des régimes apportant 2320 ou $2730 \mathrm{kcal}$ d'énergie digestible par $\mathrm{kg}$ d'aliment et ayant un rapport de 49,4 ou $58,3 \mathrm{~g}$ de protéines digestibles/1000 kcal d'énergie digestible. A l'âge de 10 semaines, ils ont été sacrifiés pour déterminer les bilans azotés et énergétiques. Une étude de la digestibilité apparente des aliments durant la troisième semaine expérimentale a confirmé que l'incorporation de paille en subsitution de l'amidon entraîne bien une dilution de la concentration en énergie digestible du régime et que les lapins sont capables d'ajuster leur consommation énergétique. Les besoins azotés ont toujours été couverts et l'azote, dans ce cas, n'a pas été un facteur limitant de la croissance. L'incorporation de paille a entraîné une modification du développement du tube digestif et de la répartition du contenu digestif.
\end{abstract}

\section{Introduction}

Le Lapin, comme de nombreux monogastriques, règle son ingestion en fonction de ses besoins en énergie (Lebas, 1975; Bombeke, Okerman \& Mormans, 1978 ; Spreadbury \& Davidson, 1978). La valeur énergétique du régime détermine donc le niveau de consommation et, par conséquent, les apports en azote et en minéraux. Cette régulation peut donc avoir une incidence sur la satisfaction des besoins autres qu'énergétiques, tels que ceux en azote, auxquels nous nous sommes plus particulièrement intéressés. Ainsi, avons-nous étudié l'influence de la concentration en énergie digestible du régime sur l'utilisation de l'azote et de l'énergie chez le Lapin en croissance. Pour cela, nous avons comparé les performances zootechniques et les bilans énergétique et azoté chez des lapins nourris avec des aliments à concentration énergétique soit faible $(2320 \mathrm{kcal} \mathrm{ED} / \mathrm{kg})$, soit élevée $(2730 \mathrm{kcal} \mathrm{ED} / \mathrm{kg})$ et dont le rapport protéines digestibles/énergie digestible (PD/ED) était de 49 ou $58 \mathrm{~g} / 1000 \mathrm{kcal}$ ED. L'analyse a porté également sur le développement du tube digestif. 


\section{1. - Matériel et méthodes}

\subsection{Les régimes (tableau 1)}

L'expérience a été réalisée selon un schéma factoriel comprenant deux niveaux d'énergie digestible (ED) 2320 et $2730 \mathrm{kcal} \mathrm{ED} / \mathrm{kg}$ d'aliment, et deux rapports protéines digestibles/énergie digestible : $\mathrm{PD} / \mathrm{ED}=49,4$ et $58,3 \mathrm{~g}$ de protéines digestibles/ $1000 \mathrm{kcal} \mathrm{ED}$ (rapports calculés à partir des deux concentrations énergétiques et d'un apport théorique de $13,5 \mathrm{~g}$ de protéines digestibles pour $100 \mathrm{~g}$ d'aliment). Ceci nous a conduit à utiliser des régimes contenant effectivement 11,5 - 12,8 - 12,7 et 14,2 p. 100 de protéines digestibles. La dilution énergétique a été obtenue par incorporation de paille en substitution de l'amidon de maïs (tableau 1). Les différentes teneurs en protéines ont été obtenues par des apports adaptés de tourteau de soja.

\subsection{Les animaux}

60 lapins de race Californienne des deux sexes, répartis en 4 lots ont été élevés de 5 à 10 semaines en cage individuelle. 28 d'entre eux ont été placés dans des cages permettant de mesurer l'utilisation digestive des différents éléments, par collecte quantitative des fèces au cours de la $8^{*}$ semaine postnatale.

\subsection{Contrôles}

Les animaux ont été nourris ad libitum et ont disposé d'eau à volonté. Pour l'ensemble des animaux, la consommation alimentaire et la croissance ont été contrôlées hebdomadairement et individuellement. Au cours des mesures de digestibilité, c'est quotidiennement que le contrôle de consommation a été réalisé ainsi que la récolte des fèces pendant 8 jours consécutifs. Les 60 animaux ont été abattus à âge constant (10 semaines) par dislocation cervicale, puis saignée. Le tube digestif a été prélevé. L'estomac et le caecum ont été isolés, pesés pleins puis vides.

\subsection{Analyses chimiques}

Les fèces ont été séchées immédiatement après récolte, à $103^{\circ} \mathrm{C}$ durant 24 heures et c'est sur le produit sec que les différentes analyses ont été effectuées après détermination de la matière sèche. C'est également sur des échantillons préalablement séchés que les aliments ont été soumis aux différentes analyses.

Toutes les analyses corporelles ont été réalisées à partir d'un échantillon de l'animal entier, saigné, vidé du contenu digestif et de l'urine. Tous les résultats tiennent compte du sang perdu (toutes les analyses ont été effectuées sur des échantillons de sang). Pour obtenir une fraction homogène du corps, nous les avons broyés au broyeur à hélice «robot-coupe », puis au hachoir «Scharfen». Environ $250 \mathrm{~g}$ de ce produit ont été congelés, puis repris au broyeur à billes «Dangoumeau » sous azote liquide et ensuite lyophilisés. La teneur en matière sèche a également été contrôlée pour ce produit. 
TABLEAU 1

Composition des régimes expérimentaux

Composition of experimental diets

\begin{tabular}{|c|c|c|c|c|}
\hline Aliments (Diets) (1) & B.B. & B.H. & H.B. & H.H. \\
\hline Teneurs théoriques (Theoretical levels) & & & & \\
\hline $\begin{array}{l}\text { 一 Energie digestible }(\mathrm{kcal} / \mathrm{kg}) \ldots \ldots \ldots \ldots \ldots \ldots \\
\text { Digestible energy }\end{array}$ & 2320 & 2320 & 2730 & 2730 \\
\hline $\begin{array}{l}\text { - Protéines digestibles }(\mathrm{g} / 100 \mathrm{~g}) \ldots \ldots \ldots \ldots \\
\text { Digestible proteins }\end{array}$ & 11,5 & 13,5 & 13,5 & 15,9 \\
\hline $\begin{array}{l}\text { - g protéines digestibles } / 1000 \mathrm{kcal} \text { E.D. } \ldots \\
\text { g digestible proteins } / 1000 \mathrm{kcal} \text { D.E. }\end{array}$ & 49,7 & 58,3 & 49,4 & 58,2 \\
\hline \multicolumn{5}{|l|}{ Composition centésimale } \\
\hline - Paille de blé $\ldots \ldots \ldots \ldots \ldots \ldots \ldots$ & 20,5 & 20,0 & 5,0 & 4,5 \\
\hline $\begin{array}{l}\text { - Tourteau de soja } 50 \ldots \ldots \ldots \ldots \ldots \ldots \ldots \\
\text { Soya meal } 50\end{array}$ & 19,0 & 24,0 & 24,0 & 30,5 \\
\hline - Amidon de maïs $\ldots \ldots \ldots \ldots \ldots \ldots \ldots \ldots$ & 18,4 & 13,9 & 28,9 & 22,9 \\
\hline $\begin{array}{l}\text { - Luzerne déshydratée }(17,5 \% \text { mat. azotées }) \\
\text { Dehydrated lucerne }(17.5 \% \text { proteins })\end{array}$ & 28,0 & 28,0 & 28,0 & 28,0 \\
\hline - $\underset{\text { Wheat }}{\text { Blé }} \ldots \cdots \cdots \cdots \cdots \cdots \cdots \cdots \cdots \cdots \cdots \cdots \cdots \cdots \cdots$ & 10,0 & 10,0 & 10,0 & 10,0 \\
\hline $\begin{aligned} \text { - C.M.V. (2) } & \text { Minerals and vitamins }\end{aligned}$ & 4,0 & 4,0 & 4,0 & 4,0 \\
\hline $\begin{array}{l}\text { dl méthionine } \ldots \ldots \ldots \ldots \ldots \ldots \ldots \ldots \ldots \\
\text { dl methionine }\end{array}$ & 0,10 & 0,12 & 0,12 & 0,14 \\
\hline \multicolumn{5}{|l|}{$\begin{array}{l}\text { Composition analytique } \\
\text { Analytical composition }\end{array}$} \\
\hline 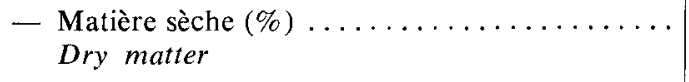 & 89,9 & 89,9 & 89,7 & 89,8 \\
\hline $\begin{aligned} & \text { - Cendres }(\% \text { M.S. }) \ldots \ldots \ldots \ldots \ldots \ldots \ldots \ldots \ldots \\
& \text { Ash }(\% \text { D.M. })\end{aligned}$ & 8,8 & 9,7 & 8,4 & 8,9 \\
\hline $\begin{array}{l}\text { - Matières azotées }(\mathrm{N} \times 6.25)(\% \text { M.S. }) \ldots \ldots \\
\text { Crude protein }(\% \text { D.M. })\end{array}$ & 16,0 & 17,2 & 17,2 & 19,2 \\
\hline $\begin{array}{l}\text { - Energie brute }(\mathrm{kcal} / \mathrm{kg}) \ldots \ldots \ldots \ldots \ldots \ldots \\
\text { Crude energy }\end{array}$ & 3670 & 3700 & 3720 & 3700 \\
\hline - Energie digestible $(\mathrm{kcal} / \mathrm{kg}) \ldots \ldots \ldots \ldots \ldots \ldots$ & 2280 & 2240 & 2610 & 2670 \\
\hline $\begin{array}{l}\text { - Protéines digestibles }(\mathrm{g} / 100 \mathrm{~g}) \ldots \ldots \ldots \ldots \ldots \\
\text { Digestible proteins }\end{array}$ & 11,5 & 12,8 & 12,7 & 14,2 \\
\hline $\begin{array}{l}-\mathrm{g} \text { protéines digestibles } / 1000 \mathrm{kcal} \text { E.D. } \ldots \\
g \text { digestible proteins } / 1000 \mathrm{kcal} \text { D.E. }\end{array}$ & 50,5 & 57,1 & 48,8 & 53,3 \\
\hline
\end{tabular}

(1) La $1^{\text {re }}$ lettre correspond à la concentration énergétique basse ou haute, la $2^{\circ}$ au rapport $\mathrm{PD} / \mathrm{ED}$, bas ou haut.

The first letter is designing the energy input level $(B=l o w, H=h i g h)$, the second one the digest. prot. / digest. energy ratio $(B=$ low, $H=$ high).

(2) Colin, Arkhurst \& Lebas (1973). 
Les cendres ont été mesurées après incinération une heure à $300{ }^{\circ} \mathrm{C}$ et 5 heures à $550^{\circ} \mathrm{C}$. Les teneurs en protéines ont été déterminées par la méthode Kjeldahl $(\mathrm{N}$ Kjeldahl $\times 6,25)$ modifiée pour dosage automatique sur « Technicon . Les teneurs en énergie ont été évaluées avec un calorimètre adiabatique Gallenkamp "Autobomb ».

\subsection{Mesures de digestibilité et bilans}

La digestibilité apparente des différents composants de la ration (matière sèche, matière organique, énergie, protéines et cendres) a été estimée par le calcul du coefficient d'utilisation digestive apparente (CUDa).

Les bilans azoté et énergétique ont été calculés par différence entre les quantités azotée et énergétique contenues dans le corps des animaux en fin d'expérience et celles existant au début, estimées à l'aide de 10 lapins témoins sacrifiés dans les mêmes conditions à l'âge de 5 semaines.

\subsection{Analyse mathématique des résultats}

Les séries de données ont été étudiées par analyse de variance, selon le modèle factoriel avec interaction (programme Cønфr du Laboratoire de Biométrie du C.R. de Toulouse).

\section{2. - Résultats}

\subsection{Quantités ingérées et performances de croissance (tableau 2)}

Comme nous l'avions prévu, la consommation a été plus faible pour les aliments riches en énergie digestible $(107 \mathrm{~g} / \mathrm{j}$ contre 119 et $124 \mathrm{~g} / \mathrm{j})$ alors que les quantités d'énergie digestible ingérées ont été identiques pour les 4 régimes $(273 \mathrm{kcal} \mathrm{ED} / \mathrm{j})$. Aussi l'ingestion de protéines digestibles a-t-elle été déterminée par le rapport $\mathrm{PD} / \mathrm{ED}$ : elle a été de 13,8 et $13,6 \mathrm{~g} / \mathrm{j}$ pour les animaux qui recevaient les aliments à $49 \mathrm{~g} \mathrm{PD} /$ $1000 \mathrm{kcal} E D$ et de $15,9-15,4 \mathrm{~g} / \mathrm{j}$ pour les animaux recevant les deux autres aliments.

Les lapins ont réalisé des croissances comparables pour les 4 lots $(35 \mathrm{~g} / \mathrm{j}$ en moyennc) malgré cette consommation légèrement différente de protéines digestibles (tableau 2).

La dilution de la concentration énergétique du régime par incorporation de paille a donc augmenté la consommation sans améliorer la croissance : aussi l'indice de consommation des aliments à $2320 \mathrm{kcal}$ ED est-il plus élevé que celui des aliments à $2740 \mathrm{kcal} \operatorname{ED}(3,56$ vs 3,23 g d'aliment/g gain de poids vif). L'efficacité alimentaire [quantité d’énergie digestible ingérée $(\mathrm{kcal}) /$ gain de poids vif $(\mathrm{g})$ ] a été identique pour les 4 lots et égale en moyenne à $81 \mathrm{kcal} / \mathrm{g}$. Exprimée en protéines digestibles ingérées rapportées au gain de poids vif $(\mathrm{g} / \mathrm{g})$, elle a varié dans le même sens que les quantités de protéines ingérées, mais de façon non significative $(0,39$ et 0,41 pour les régimes à $49 \mathrm{~g}$ de $\mathrm{PD} / 1000 \mathrm{kcal} \mathrm{ED}$ contre 0.45 pour les régimes à $56 \mathrm{~g}$ de $\mathrm{PD} / 1000 \mathrm{kcal}$ ED). 


\section{Tableau 2}

Quantités ingérées, croissance et efficacité alimentaire

Daily feed intake, growth and feed conversion ratios

\begin{tabular}{|c|c|c|c|c|c|c|c|}
\hline \multicolumn{2}{|c|}{ Régimes (Diets) } & \multirow{2}{*}{$\begin{array}{l}\text { B.B. } \\
808\end{array}$} & \multirow{2}{*}{$\begin{array}{l}\text { B.H. } \\
842\end{array}$} & \multirow{2}{*}{$\begin{array}{l}\text { H.B. } \\
824\end{array}$} & \multirow{2}{*}{$\frac{\text { H.H. }}{810}$} & \multirow{2}{*}{ 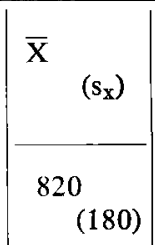 } & \multirow{2}{*}{$\begin{array}{l}\begin{array}{c}\text { Signifi- } \\
\text { cation } \\
\text { statis- } \\
\text { tique (1) }\end{array} \\
\text { NS }\end{array}$} \\
\hline $\begin{array}{l}\text { Poids } \\
\text { moyen }(\mathrm{g})\end{array}$ & $\begin{array}{l}\text { initial } \\
\text { initial }\end{array}$ & & & & & & \\
\hline $\begin{array}{l}\text { Mean budy } \\
\text { weight }(g)\end{array}$ & $\begin{array}{l}\text { final } \ldots \ldots \ldots \\
\text { at slaughter }\end{array}$ & 2036 & 2083 & 1974 & 2020 & 2029 (279) & NS \\
\hline \multicolumn{2}{|c|}{$\begin{array}{l}\text { Consommation d'aliment }(\mathrm{g} / \mathrm{j}) \ldots \\
\text { Daily feed intake }(g / d)\end{array}$} & $\begin{array}{l}119^{a} \\
(2)\end{array}$ & $124^{a}$ & $107 \mathrm{~b}$ & $108^{\mathrm{b}}$ & 115 (18) & $\begin{aligned} * * & (\mathrm{ED}) \\
& (D E)\end{aligned}$ \\
\hline \multicolumn{2}{|c|}{$\begin{array}{l}\text { Gain de poids }(\mathrm{g} / \mathrm{j}) \\
\text { Daily weight gain }(g / d)\end{array}$} & 35,7 & 35,8 & 33,4 & 34,6 & $\begin{array}{r}35,0 \\
(5,9)\end{array}$ & NS \\
\hline \multicolumn{2}{|c|}{$\begin{array}{l}\text { Indice de consommation } \\
\quad \text { (ingéré g / gain de poids g) } \ldots . \\
\text { Feed conversion ratio }\end{array}$} & $3,52: 4$ & $3,60^{\mathrm{a}}$ & $3,26^{b}$ & $3,20^{\mathrm{b}}$ & $\begin{array}{l}3,40 \\
(0,47)\end{array}$ & $\begin{aligned} * & (\mathrm{ED}) \\
& (D E)\end{aligned}$ \\
\hline \multicolumn{8}{|c|}{$\begin{array}{l}\text { Energie digestible ingérée } \\
\text { Digestible energy intake }\end{array}$} \\
\hline \multicolumn{2}{|c|}{$-\underset{k c a l / j}{\mathrm{k} c a l / d}(\ldots \ldots \ldots \ldots \ldots \ldots$} & 253 & 278 & 280 & 289 & $273(40)$ & NS \\
\hline \multicolumn{2}{|c|}{$\begin{array}{c}-\mathrm{kcal} / \mathrm{g} \text { de gain } \ldots . . . . . . . . \\
\mathrm{kcal} / \mathrm{g} \text { of live weight gain }\end{array}$} & 7,7 & 7,9 & 8,5 & 8,5 & $\begin{array}{c}8,1 \\
(1,2)\end{array}$ & NS \\
\hline \multicolumn{2}{|c|}{$\begin{array}{l}\text { Protéines digestibles ingérées } \\
\text { Digestible protein intake } \\
-\mathrm{g} / \mathrm{j} \ldots \ldots \ldots \ldots \ldots \ldots \\
\quad \text { g/day }\end{array}$} & 13,8 & 15,9 & 13,6 & 15,4 & $\begin{array}{r}14,62 \\
(2,2)\end{array}$ & NS \\
\hline \multicolumn{2}{|c|}{$\begin{array}{c}\mathrm{g} / \mathrm{g} \text { de gain } \ldots \ldots \ldots \ldots \\
g / g \text { of live weight gain }\end{array}$} & 0,39 & 0,45 & 0,41 & 0,45 & $\begin{array}{l}0,42 \\
(0,06)\end{array}$ & NS \\
\hline
\end{tabular}

(1) NS : Non significatif.

Non significant.

***: $: \mathrm{p}<0,001$.

$* *: \mathrm{p}<0,01$.

$*: \mathrm{p}<0,05$.

(2) $a, b$ : Les valeurs ayant en indice une lettre différente diffèrent au seuil $p=0,05$.

On the same line indicate significant differences for $p=0.05$. 
Tableau 3

Coefficients d'utilisation digestive des principaux constituants des aliments Digestibility of the diets

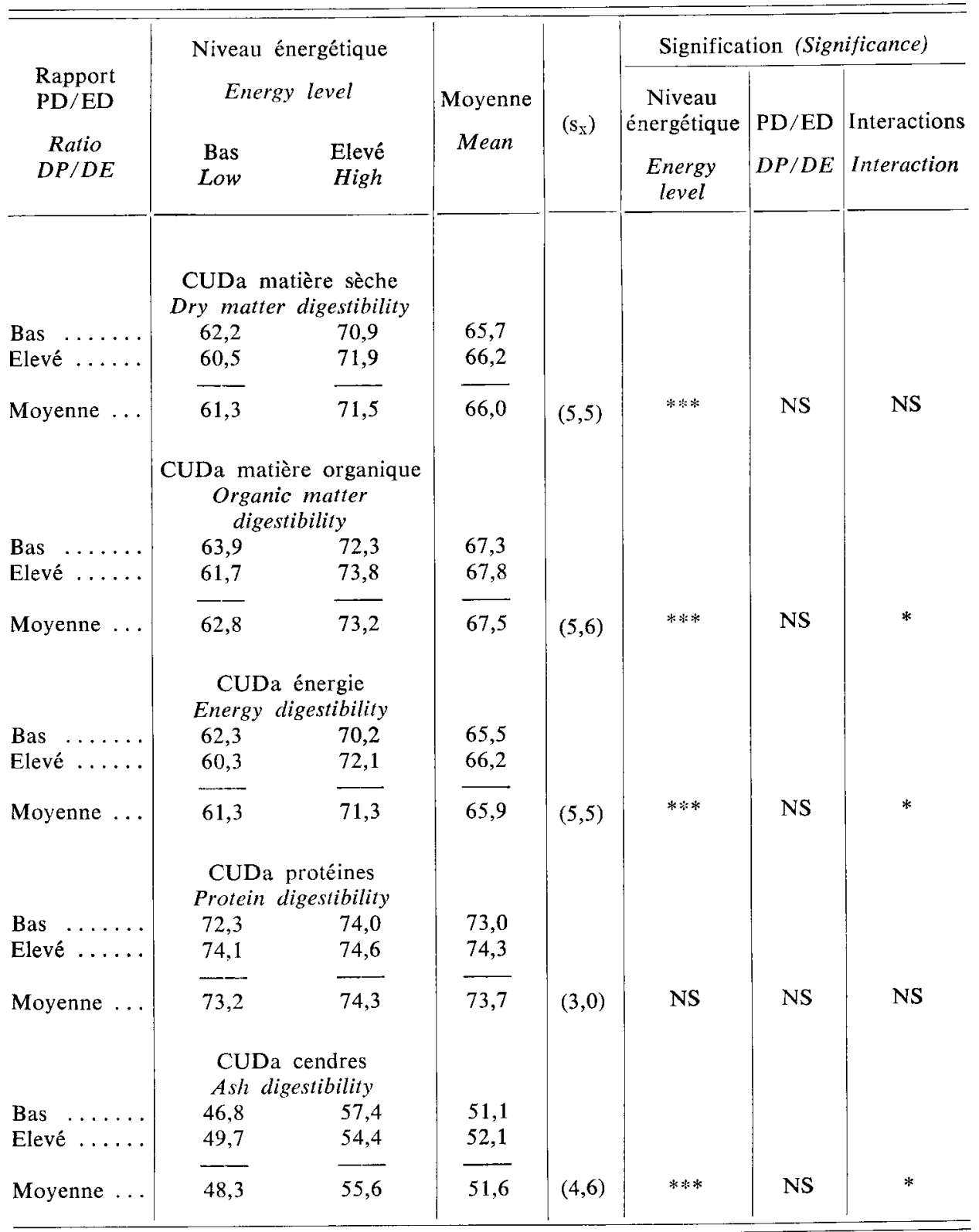




\section{TABLEAU 4}

Utilisation métabolique de l'azote et de l'énergie

Efficiency of protein and energy deposition

\begin{tabular}{|c|c|c|c|c|c|c|}
\hline Régimes (Diets) & B.B. & B.H. & H.B. & H.H. & $\left(\mathrm{s}_{\mathrm{x}}\right)$ & $\begin{array}{l}\text { Signification } \\
\text { statistique }\end{array}$ \\
\hline $\begin{array}{l}\text { Protéines retenues }(\mathrm{g}) \\
(\mathrm{N} \times 6,25) \\
\text { Protein deposition }\end{array}$ & & & & & & \\
\hline $\begin{aligned} \text { - Période totale } \ldots \ldots \ldots \ldots \\
\\
\text { Total period }\end{aligned}$ & 268 & 265 & 264 & 274 & $268(45)$ & NS \\
\hline - Par jour ................. & 7,67 & 7,56 & 7,53 & 7,82 & $\begin{array}{l}7,65 \\
(1,29)\end{array}$ & NS \\
\hline $\begin{array}{l}\text { Rétention azotée }(\%) \ldots \ldots \\
N \text { retained as percent of di- } \\
\text { gest } N\end{array}$ & $55,4^{a}$ & $47,7 \mathrm{~b}$ & $55,7^{a}$ & $50,7^{\mathrm{b}}$ & $\begin{array}{r}52,6 \\
(7,3)\end{array}$ & $\begin{aligned} * * & \text { PD/ED } \\
& D P / D E\end{aligned}$ \\
\hline $\begin{array}{l}\text { C.U.P. azoté }(\%) \ldots \ldots \ldots \\
N \text { retained as percent of } N \text { in- } \\
\text { take }\end{array}$ & $40,1^{\mathrm{a}}$ & $35,5^{b}$ & $41,2^{a}$ & $37,6^{\mathrm{b}}$ & $\begin{array}{r}38,7 \\
(5,3)\end{array}$ & ** $\begin{aligned} & \mathrm{PD} / \mathrm{ED} \\
& D P / D E\end{aligned}$ \\
\hline $\begin{array}{l}\text { Energie retenue (kcal) } \\
\text { Energy deposition }\end{array}$ & & & & & & \\
\hline $\begin{array}{l}\text { - Période totale } \ldots \ldots \ldots \ldots \\
\text { Total period }\end{array}$ & 2420 & 2300 & 2430 & 2510 & 2410 & NS \\
\hline $\begin{array}{l}\text { - Par jour .................. } \\
\text { Daily deposition rate }\end{array}$ & 69,1 & 65,7 & 69,3 & 71,6 & $\begin{array}{c}69,0 \\
(17,0)\end{array}$ & NS \\
\hline $\begin{array}{l}\text { Rétention énergie }(\%) \ldots . . . \\
\text { Energy deposition as percent of } \\
\text { D.E. intake }\end{array}$ & 25,1 & 23,5 & 24,7 & 24,6 & $\begin{array}{l}24,5 \\
(4,5)\end{array}$ & NS \\
\hline $\begin{array}{l}\text { C.U.P. énergie }(\%) \ldots \ldots \ldots \\
\text { Energy deposition as percent of } \\
\text { crude energy intake }\end{array}$ & $15,6^{\mathfrak{a}}$ & $14,2^{\mathrm{a}}$ & $17,4^{b}$ & $17,7^{b}$ & $\begin{array}{r}16,2 \\
(3,3)\end{array}$ & ** $\mathrm{ED}$ \\
\hline
\end{tabular}

\subsection{Digestibilité des différents constituants de la ration (tableau 3)}

L'incorporation de la paille a bien provoqué une diminution de la digestibilité globale de la ration : nous avons noté une diminution de 13 p. 100 du coefficient d'utilisation digestive apparente (CUDa) de la matière sèche, de la matière organique, de l'énergie et des cendres. Par contre, l'utilisation digestive de l'azote ne s'est pas révélée significativement différente pour les 4 traitements (CUDa $=74$ p. 100). 
La modification du rapport $\mathrm{PD} / \mathrm{ED}$ n’a provoqué de variation significative pour aucun des nutriments étudiés.

\subsection{Protéines et énergie fixées (tableau 4)}

Les quantités d'énergie fixées ont été identiques dans les 4 lots : elles ont été de $69 \mathrm{kcal}$ par jour. Les animaux ont retenu des quantités de protéines également comparables (7,69 g par jour). Ceci s'explique par une rétention azotée plus faible de 13 p. 100 lorsque les animaux ont ingéré plus de protéines digestibles, c'est-à-dire pour un rapport $\mathrm{PD} / \mathrm{ED}$ de l'aliment élevé. Comme il n'y a pas eu de différence au niveau de l'utilisation digestive de l'azote (tableau 3), le coefficient d'utilisation digestive pratique de l'azote a varié dans le même sens que la rétention (tableau 4).

Le rendement de l'utilisation de l'énergie digestible ( $E$ fixée/ $E$ digérée) a été identique pour les 4 lots : il a été en moyenne de 24,5 p. 100 . La fraction d'énergie ingérée, qui a été retenue par l'animal (CUP), a donc été fonction uniquement de l'utilisation digestive de cette énergie.

\subsection{Composition corporelle (tableau 5)}

Le poids moyen d'abattage a été comparable pour les 4 lots (en moyenne de $2,0 \mathrm{~kg}$ ). Les animaux recevant les régimes concentrés en énergie digestible ont retenu moins d'eau que les animaux des deux autres lots $(67$ p. 100 contre 69 p. 100 - tableau 5). Les teneurs en minéraux et en protéines de la masse corporelle n'ont pas été significativement différentes. Les animaux recevant l'aliment renfermant $2730 \mathrm{kcal}$ ED par $\mathrm{kg}$, ont déposé légèrement plus de gras que les animaux des autres lots : en effet, le pourcentage de lipides estimé est de 24,4 p. 100 pour les premiers contre 23,1 p. 100 (tableau 5).

\section{TABLEAU 5}

Composition corporelle des animaux

Whole body composition

\begin{tabular}{|c|c|c|c|c|c|c|}
\hline \multirow{2}{*}{$\begin{array}{l}\text { Régimes (Diets) } \\
\text { ED }(\mathrm{kcal} / \mathrm{kg}) \ldots \ldots \ldots \ldots \ldots \\
D E(k c a l / k g) \\
\mathrm{PD} / \mathrm{ED}(\mathrm{g} / 1000 \mathrm{kcal}) \ldots \ldots \\
D P / D E\end{array}$} & \multicolumn{2}{|c|}{2320} & \multicolumn{2}{|c|}{2730} & \multirow[b]{2}{*}{$\left(s_{x}\right)$} & \multirow{2}{*}{$\begin{array}{l}\text { Signification } \\
\text { statistique }\end{array}$} \\
\hline & 49 & 56 & 49 & 56 & & \\
\hline $\begin{array}{l}\text { Matière sèche }(\%) \\
\text { Dry matter }(\%)\end{array}$ & 31,43 & 30,71 & 33,32 & 32,38 & $\begin{array}{c}31,93 \\
(3,03)\end{array}$ & $*$ (ED) (DE) \\
\hline $\begin{array}{l}\text { Cendres (\% MS) } \ldots \ldots \ldots \ldots \\
\text { Ash }(\% \quad D M)\end{array}$ & 12,2 & 12,3 & 12,2 & 11,5 & $\begin{array}{r}12,0 \\
(1,5)\end{array}$ & NS \\
\hline $\begin{array}{l}\text { Protéines }(\mathrm{N} \times 6,25)(\% \text { MS }) \\
\text { Crude protein }\left(\begin{array}{ll}\% & D M\end{array}\right)\end{array}$ & 64,3 & 65,0 & 63,4 & 64,2 & $\begin{array}{r}64,3 \\
(3,4)\end{array}$ & NS \\
\hline $\begin{array}{l}\text { Lipides (\% MS) (estimé) } \ldots \\
\text { Lipids }(\% \quad \text { DM) }(\text { calculated })\end{array}$ & 23,5 & 22,7 & 24,4 & 24,3 & - & - \\
\hline $\begin{array}{l}\text { EB (kcal/kg MS) } \ldots \ldots \ldots \ldots \\
\text { Crude energy }(k c a l / k g \quad D M)\end{array}$ & 5560 & 5520 & 5580 & 5630 & $\left|\begin{array}{rl}5 & 570 \\
(230)\end{array}\right|$ & NS \\
\hline
\end{tabular}




\subsection{Développement du tube digestif (tableau 6)}

A l'abattage, nous n'avons pas observé de différences significatives des poids du tube digestif et de son contenu entre les animaux des 4 lots. La masse viscérale du tube digestif est d'environ $150 \mathrm{~g}$, celle du contenu de $220 \mathrm{~g}$ en moyenne.

Cependant le poids de l'estomac et du caecum a varié avec la teneur en énergie digestible du régime. Ainsi pour les aliments à $2320 \mathrm{kcal} \mathrm{ED} / \mathrm{kg}$, c'est-à-dire riches en paille, le poids de l'estomac a été supérieur de 30 p. 100 et celui du caecum inférieur de 18 p. 100 aux poids de ces mêmes organes prélevés sur les animaux qui recevaient les régimes à $2730 \mathrm{kcal} \mathrm{ED/kg}$ (tableau 6).

\section{TABleau 6}

Poids de l'estomac et du caecum et de leur contenu

Weight of the stomach, the caecum and their content

\begin{tabular}{|c|c|c|c|c|c|c|}
\hline Régimes (Diets) & B.B. & B.H. & H.B. & H.H. & $\begin{array}{ll}X & \\
& \left(s_{x}\right)\end{array}$ & $\begin{array}{l}\text { Signification } \\
\text { statistique }\end{array}$ \\
\hline $\begin{array}{l}\text { Poids vif abattage }(\mathrm{g}) \ldots \ldots \ldots \\
\text { Slaughter weight }\end{array}$ & 2036 & 2083 & 1974 & 2020 & 2029 & NS \\
\hline $\begin{array}{l}\text { Estomac vide }(\mathrm{g}) \ldots \ldots \ldots \ldots \\
\text { Empty stomach }\end{array}$ & $22,3^{\mathrm{a}}$ & $22,6^{\mathrm{a}}$ & $19,3^{b}$ & $19,6^{b}$ & $\begin{array}{l}21,0 \\
(3,1)\end{array}$ & $\begin{aligned} & * * *(\mathrm{ED}) \\
&(D E)\end{aligned}$ \\
\hline $\begin{array}{l}\text { Contenu estomac (g) } \ldots \ldots \ldots \\
\text { Stomach content }\end{array}$ & $72,0^{\mathrm{a}}$ & $69,1^{a}$ & $53,7^{b}$ & $54,9 \mathrm{~b}$ & $\begin{array}{l}63,1 \\
(27,7)\end{array}$ & $\begin{array}{r}*(\mathrm{ED}) \\
(D E)\end{array}$ \\
\hline $\begin{array}{l}\text { Caecum vide }(\mathrm{g}) \ldots \ldots \ldots \ldots \\
\text { Empty caecum }\end{array}$ & $29,3 \mathrm{a}$ & $30,4^{a}$ & $38,1^{b}$ & $34,7 \mathrm{~b}$ & $\begin{array}{l}32,8 \\
(10,1)\end{array}$ & $\begin{aligned} * * & (\mathrm{ED}) \\
& (D E)\end{aligned}$ \\
\hline $\begin{array}{l}\text { Contenu caecum }(\mathrm{g}) \ldots \ldots \\
\text { Caecum content }\end{array}$ & 96,3 & 96,5 & 105,1 & 103,0 & $\begin{array}{l}100,0 \\
(22,3)\end{array}$ & NS \\
\hline Estomac vide & $78,2^{\mathfrak{a}}$ & $76,2^{a}$ & $56,9 \mathrm{~b}$ & $57,9^{b}$ & 68,0 & $* * *$ (ED) \\
\hline $\begin{array}{l}\text { Caecum vide } \\
\frac{\text { Empty stomach }}{\text { Empty caecum }} \times 100 \ldots \ldots\end{array}$ & & & & & $(18,3)$ & \\
\hline
\end{tabular}

Nous avons noté également des écarts au niveau des poids des contenus digestifs de ces deux compartiments; ils varient dans le même sens mais de façon moins marquée que les poids des viscères : les animaux recevant les aliments renfermant $2320 \mathrm{kcal} \mathrm{ED} / \mathrm{kg}$ ont des contenus stomacaux plus lourds $(+15 \mathrm{p} .100)$ et à l'inverse des contenus caecaux plus légers $(-7$ p. 100) que les animaux qui recevaient les régimes à $2730 \mathrm{kcal} \mathrm{ED/kg}$ (tableau 6). 


\section{3. - Discussion}

3.1. - Les aliments utilisés pour l'expérience ont eu des teneurs en énergie digestible plus faibles (de 2 à 4 p. 100) que celles qui avaient été calculées. Cependant l'écart des concentrations énergétiques est du même ordre de grandeur : 14,4 p. 100 pour 15,0 p. 100 théoriques. Les rapports $\mathrm{PD} / \mathrm{ED}$ ne se sont écartés des valeurs calculées que d'environ 2 p. 100 à l'exception de l'aliment à forte concentration en énergie digestible et à rapport $\mathrm{PD} / \mathrm{ED}$ élevé qui a un rapport $\mathrm{PD} / \mathrm{ED}$ plus faible que prévu de 8,5 p. 100 : ceci réduit l'écart entre les deux rapports $P D / E D$ haut et bas de 15,4 p. 100 à 7,4 p. 100 . Toutefois, l'analyse statistique a tout de même été réalisée en respectant le schéma factoriel initial.

3.2. - L'utilisation de lazote a été identique dans les 4 lots, quelle qu'ait été la concentration énergétique du régime, mais aussi quelle qu'ait été la teneur en protéines digestibles, parce que les besoins des animaux ont toujours été satisfaits. L'azote supplémentaire ingéré par les animaux qui recevaient $58 \mathrm{~g}$ de protéines digestibles pour $1000 \mathrm{kcal}$ d'ED a été éliminé ; en effet la rétention azotée a été plus faible de 13 p. 100 pour ces animaux.

Ouhayoun, Delmas \& Lebas (1979), en comparant des régimes riches en énergie digestible (environ $2500 \mathrm{kcal} \mathrm{ED} / \mathrm{kg}$ d'aliment), ont noté également que l'efficacité de transformation des protéines est meilleure avec un aliment à 13 p. 100 de protéines quavec un aliment à 16 p. 100.

Un apport quotidien de $14 \mathrm{~g}$ de protéines digestibles est donc suffisant pour une croissance de $35 \mathrm{~g} /$ jour, ce qui correspond à un taux de $17 \mathrm{p} .100$ de protéines pour un aliment à concentration énergétique élevée $(2740 \mathrm{kcal} \mathrm{ED} / \mathrm{kg})$ ou à un taux de 16 p. 100 pour un aliment à concentration énergétique de $2320 \mathrm{kcal} / \mathrm{kg}$. Ces résultats sont en bon accord avec les recommandations du NRC qui propose un taux de 15 à 16 p. 100 de protéines pour une concentration d'énergie digestible de $2500 \mathrm{kcal} / \mathrm{kg}$ d'aliment. Cependant, une réduction de ce taux de 15-16 p. 100 à 13-14 p. 100 pour une teneur en énergie digestible de $2600-2700 \mathrm{kcal} E D / \mathrm{kg}$ entraîne une diminution de la vitesse de croissance (Ouhayoun \& Delmas, 1980). Lanari, Parigibini \& ChieRICATO (1972) ont observé également pour des régimes à très haut niveau énergétique et pour une teneur de 19 à 20 p. 100 de protéines une baisse du gain en azote lorsque la concentration énergétique passait de $1783 \mathrm{kcal} \mathrm{EN} / \mathrm{kg}$ MS à $2260 \mathrm{kcal} \mathrm{EN} / \mathrm{kg} \mathrm{MS}$.

3.3. - La vitesse de croissance n`a pas été affectée par la dilution énergétique de laliment par incorporation de paille. La diminution de la digestibilité globale de la ration a été compensée par l'augmentation du niveau d'ingestion : il y a eu maintien de lapport d'énergie digestible à $273 \mathrm{kcal} /$ jour. L'aptitude du Lapin à régler son ingestion en fonction de ses besoins en énergie est un fait bien connu. Nos résultats confirment ceux de nombreux auteurs, et dont les plus récents montrent que la vitesse de croissance n'est pas modifiée avec des aliments riches en éléments peu digestibles, jusqu'à une teneur de 20 p. 100 de l'aliment en glucides pariétaux (LEBAS, 1975 ; Bombeke, Okerman \& Mormans, 1978 ; Spreadbury \& Davidson, 1978). Bien que les rétentions énergétiques aient été identiques, il semble que l'énergie absorbée n’a pas été utilisée de manière comparable, puisque les aliments à niveau énergétique élevé semblent induire une plus faible rétention d'eau et corrélativement une fixation de gras plus élevée. 
Il convient par ailleurs de souligner l'absence d'effet significatif dû au rapport $\mathrm{PD} / \mathrm{ED}$ sur les performances de croissance. Par contre, il est apparu une interaction des deux facteurs : niveau énergétique et rapport $\mathrm{PD} / \mathrm{ED}$ sur les coefficients d'utilisation digestive apparente (CUDa) de la matière organique, de l'énergie et des cendres. Cette interaction est difficile à expliquer sur le plan biologique; d'un point de vue statistique, il peut s'agir d'un artéfact. L'effet du niveau énergétique est très élevé et semble se répercuter jusque dans cette interaction. Ainsi, les coefficients d'utilisation digestive de la matière organique et de l'énergie varient dans le même sens que la teneur réelle en énergie digestible des aliments.

3.4. - La modification du développement du tube digestif, notamment les écarts de poids de l'estomac et du caecum et la répartition du contenu digestif, avec le niveau énergétique de la ration peut traduire une adaptation du mode de fonctionrement du tube digestif. La vitesse de transit peut être affectée : ainsi, LEBAS \& LAPLACE (1977) émettent l'hypothèse selon laquelle l'augmentation du taux des constituants de la paroi végétale entraîne une accélération du transit, provoquant consécutivement une diminution de la digestibilité globale de la ration. La revalorisation par la caecotrophie d'une fraction des nutriments s'exerce-t-elle dans les mêmes proportions dans le cas des régimes dilués en énergie que dans celui des régimes riches en énergie? Pour répondre à cette question, nous avons procédé à des mesures de production de caecotrophes sur des animaux recevant des régimes isoazotés et à 2320 ou $2730 \mathrm{kcal} \mathrm{ED} / \mathrm{kg}$ d'aliment. L'excrétion des caecotrophes exprimée en matière fraîche ou en matière sèche, a été identique pour les deux régimes. Les différences de poids des contenus de l'estomac et du caecum ne sont donc pas dues à une variation entre régimes des quantités recyclées par caecotrophie.

\section{Conclusion}

Cette expérimentation nous a permis de vérifier que les lapins sont capables d'ajuster leur consommation énergétique lorsque les aliments apportent 2240 à $2670 \mathrm{kcal}$ d'énergie digestible par $\mathrm{kg}$.

Les besoins azotés se trouvent couverts pour ces deux concentrations en énergie lorsque le rapport protéines digestibles/énergie digestible (PD/ED) varie de 48,8 à 57,1. Dans ces limites, le facteur protéine ne s'est donc pas révélé comme facteur limitant de la croissance.

Cependant, d'autres auteurs ont trouvé, à des niveaux énergétiques plus élevés, une influence du taux azoté sur la croissance. Dans notre expérience, les niveaux azotés étaient trop élevés. Ces travaux méritent donc d'être poursuivis, avec des régimes moins riches en protéines pour définir les besoins azotés pour la croissance et trouver l'équilibre azote-énergie optimal. Le fait que le Lapin règle son ingestion sur ses besoins en énergie n'est donc pas un inconvénient en soi pour la nutrition azotée si les taux d'azote sont suffisamment importants. 


\section{Summary}

Balance between protein and energy supplies in growing rabbit feeding

Sixty 5-week old rabbits of both sexes were offered four diets with a digestible energy of $2320 \mathrm{kcal} / \mathrm{kg}$ or $2730 \mathrm{kcal} / \mathrm{kg}$ and 49.3 or $58.3 \mathrm{~g}$ digestible protein $/ 1000 \mathrm{kcal}$ digestible energy. At the end of the 5-week experimental period, the animals were slaughtered to determine the energy and protein balances. Control of digestibilities was made during the third experimental week. Replacement of starch by straw decreased the digestible energy content of the diets, but the animals kept their daily digestible energy intake fairly constant. There was no difference in growth performance and nitrogen balance. In this study, the proteins were not a limiting factor for growth and protein deposition. Replacement of starch by straw induced gut development changes and also a different distribution of digesta.

\section{Références bibliographiques}

Bombere A., Okerman F., Mormans R., 1978. L'influence de la granuiation à sec et à la vapeur des rations à teneurs différentes en énergie sur les résultats de production des lapins de chair. Revue de l'Agriculture, 31, 945-955.

Colin M., Arkhurst G., Lebas F., 1973. Effet de l'addition de méthionine au régime alimentaire sur les performances de croissance chez le Lapin. Ann. Zootech., 22, 485492.

Lanari D., Parigi-Bini R., Chiericato G.M., 1972. Effecto della grassatura e di diversi rapporti energie/proteine della dieta sulla composizione della carcasse di conigli da carne. Rivista di zootecnia, 45, 337-348.

LEBAS F., 1975. Influence du niveau énergétique de l'aliment sur les performances de croissance chez le Lapin. Ann. Zootech., 24, 281-288.

Lebas F., Laplace J.P., 1977. Growth and digestive transit in the rabbit. Variations determined by physical form, composition and crude fiber content of the feed. Ann. Biol. anim. Biochim. Biophys., 17, 535-538.

Ouhayoln J., DElmas D., Lebas F., 1979. Note: Influence du taux protéique de la ratica sur la composition corporelle du Lapin. Anr. Zootech., 28, 453-458.

Oukayoun J., Delmas D., 1980. Influence du niveau protéique du régime sur le développement corporel de lapins Néo-Zélandais. Mémoire II Congrìs Mondial de Cuniculture. Barcelone, avril 1980, vol. 2, 93-100.

Spreadbury D., Davidson J., 1978. A study of the need for fibre by the growing NewZeland White Rabbit. J. Sci. Food Agric., 29, 640-648. 